\title{
Re-Oreintation of Nigerians Towards Mental Health: Its Counseling Implications
}

\author{
Dr. (Mrs.) Bukoye Roseline Olufunke \\ Department of Counseling Psychology, Ibrahim Badamasi Babangida (IBB) \\ University, Niger State, Nigeria
}

doi: 10.19044/esj.2017.v13n17p302 URL:http://dx.doi.org/10.19044/esj.2017.v13n17p302

\begin{abstract}
Mental health is a state of well-being which allows a better realisation of one's own potentials. With good mental health, individuals are able to cope productively with life situations. According to WHO (2002), hundreds of millions of people worldwide are affected by mental health problems. This led to their defection in terms of behavioural, neurological, physical, emotional, and substance use. It was discovered that about 36 million people worldwide are HIV positive, and about 20 million people have died from AIDS, tobacco, alcohol, amongst others. The use of hard drugs are discovered as potential addictive substance that have led to major health problems like heart diseases, stroke, cancer, liver diseases, fever, amongst others. Mental illness/problem occurs due to careless attitudes towards mental health education. Most middle and low income countries devote less than $1 \%$ of their health expenditure to mental health and mental health education thereby worsening the health condition of the citizens. This paper, therefore, sees it as point of urgency to re-orientate Nigerians towards mental health and its counseling implications.

For its effectiveness, the roles of counseling cannot be over- emphasised. There is need for counsellors to inculcate into the public mental health skills, self-management skills, and self-descriptive culture through seminars and workshops. The Counsellor should bring to the awareness of the public information about their lifestyles regarding sleeping procedures, eating behaviour, nutrition, exercise, and stress management through enlightenment campaigns and medical programmes. Other recommendations include; the government in collaboration with the counsellors and NGOs should embark on comprehensive mental health problems preventive programmes. Also, mental health policies, legislation, community care giver facilities, and treatments for people with mental illness should be given proper attention.
\end{abstract}

Keywords: Re-Orientation, Nigerians, Towards, Mental, Health Counseling 


\section{Background of study}

It is a fact that people who have no mental symptoms are not necessarily physically healthy. For example, overworked and active individuals may feel good. Consequently, they may not have the capacity to do like those who are functioning with their day to day activities without any sign of emotional and psychological problems. Also, they may still have some necessary characteristics that well-adjusted people possess. In a simple statement, it means nobody is totally mentally healthy.

On the 9th of October 2008, the WHO launched its action programme in Geneva. The Mental Gap Action Programme (MGAP) is aimed at scaling up services for mental, neurological, and substance disorders for countries especially with low and middle income. According to WHO (2001), the programme asserts that with proper care, psychosocial assistance and medication, majority of people could be treated for depression, schizophrenia, and epilepsy. Thus, this would prevent any form of suicide and also assist people to live a normal life even where resources are scarce.

The definition of mental health considered the welfare of the people as equally very important. A mentally healthy person is able to meet the challenges of development in maintaining a satisfying sense of personal identification. Healthy individuals are able to adjust to their environment. Consequently, wellness must include external behaviour, inner feelings, behaviour from others, and the positive assessment of individual by the society. For example, the fact a drug dealer, thief, a prostitute, cultist, among others have adequate and adjustable behaviour and enjoys feeling of happiness does not mean such individual is healthy at all. This is because he or she has deviated from societal norms and has broken the law.

John (2012) in his study stated that the social and individual welfare have to be satisfied in mental health to enhance a better life adjustment. A healthy individual adjusts to life by performing his personal and societal roles productively. However, these are indicators to optimum growth and happiness. Attitudes vary greatly; therefore, life would be simple, if nothing stands in the way of meeting one's needs.

A healthy person thinks clearly, likes his/herself, and is able to solve the problems he/she encounters. This simply means that such individual has good mental health. Sometimes, some people do not think clearly. Thus, they find it even very difficult to get a clue about their problems. These types of people may need help with their mental health.

\section{Concept of Mental Health}

According to WHO (2014), mental health is a state of well-being in which every individual realizes his or her own potentials, able to cope with the normal stresses of life, work productively, think fruitfully, and are able to 
make useful contributions within his/her community. WHO (2016) went further to emphasize that, mental health is a state of complete physical, emotional, psychological, personal and social well-being, and not merely the absence of diseases or infirmity. From the United Nation (UN) Sustainable Development Goal (2015) in promoting mental health and well-being, the prevention and treatment of substance abuse are integral parts of the sustainable development agenda to transform the World by 2030. However, this was adopted by the United Nations General Assembly on 25th September, 2015. This is in recognition of the importance of the areas of health within global development and health priorities.

A mentally healthy person is one who is functioning at a high level of behavioural, emotional adjustment and adaptiveness, and not one who is simply not ill. Mental health from the researcher's point of view is the ability to face the real facts of life and adapt to it in order to gain the greatest possible satisfaction. Usually, there are obstacles in achieving the greatest possible satisfaction in life; such obstacles may be a challenge to an individual or a roadblock. Where such cannot be overcome, then the emotional needs may be difficult to meet. Therefore, such an individual may become angry or hostile, or may feel fearful or anxious and eventually adopts defense mechanisms.

Mental health is the emotional, social, and intellectual fitness of the mind and body (Patel, 2013). He further stressed that one sign of mental health is the ability to find satisfaction in relationship with others such as friends, family, and co-workers. Good mental health brings about positive co-existence which enhances positive interpersonal relationship leading to peace of mind and balanced emotions. This is what every individual needs in order to be adequately adjusted to life situations and assurance to good health. In order to help your positive thinking and comfort of mind, look for good mental health.

\section{Prevalence of Mental Health Problems}

Mental health problems which include neurological disorder, behaviour disorders, among others are common to all countries and cause immense suffering. People with these disorders, according to Okpenge (2008), are often subjected to social isolation, poor quality of life, and increased mortality. These disorders, as he said, are the causes of a staggering economy. From WHO (2002), over one hundred million people worldwide are affected by mental illness, behavioural, neurological and substance use disorders. For instance, an estimate made by WHO (2002) showed that 154 million people globally suffer from depression, 25 million People from schizophrenia, 15 million people from drug use disorder, and 100 million are affected by alcoholic use disorder. A recently published 
WHO reports showed that 50 million people suffer from epilepsy and 24 million from asthma and other serious diseases. WHO (2006) study showed that in 2005, 326 million people suffered from migraine, 61 million from cerebrovascular disorders, and 18 million suffered from neuro-infections or neurological sequence of infections. Also, according to Lewls (2011), 10 million people had disorders which required holistic remediation. Kleinman (2013) opined that people with mental health problems or illness were affected and are still affected by chronic conditions such as cancer, heart and cardiovascular diseases, diabetes, and HIV/AIDs. When untreated, they bring about unhealthy behaviour, non-compliance with prescribed medical treatments, diminished immune functioning, and poor prognosis. According to Patel (2013), cost effective treatments are available for most of these disorders. If correctly applied, it could allow most of the affected people to become well functional members of the society.

Furthermore, it was estimated that as much as $25 \%$ of all primary care consultations have a mental health component (Goldberg \& Huxley, 1992; WHO, 2006). The following are rapidly increasing: mental health problems from poverty, marginalization, poor environmental sanitation, social disadvantage, and relationship issues such as divorce; physical conditions problems such as heart disease and reductions in economic productivity; and interruption of child and adolescent educational problems, among others. Kessler (2013) stated that at the developmental level, at least $10 \%$ of children are considered to have mental health problems. With mental disorders contributing to an average $20 \%$ of disabilities at the global society level, it poses major challenges to global health. About $35-50 \%$ of mental health cases in developed countries and approximately $75-85 \%$ in less developed countries received no treatment in their mental health related problems due to the high prevalence of mild and sub-threshold cases (WHO, World Mental Health Survey Consortium (2004), and WHO, 2010). These milder cases, which can be found in various communities all over the world, require careful considerations because they are prone to progress to serious mental disorders when immediate attention is lacking.

\section{Characteristics of Mental Health Person}

John (2014) mentioned the under-listed as the characteristics of mental healthy persons.

- $\quad$ He said the mentally healthy person;

- Accepts problem and conflicts and works through them to a satisfactory end.

- $\quad$ Is able to love and accept the love of others; finds satisfaction in human relationships.

- $\quad$ Can work and play cooperatively in give and take relationships. 
- $\quad$ Tries to understand the reasons for his/her own behaviour and the behaviour of others. Remembers that behaviour is an effort to satisfy emotional and physical needs.

- $\quad$ Uses defense mechanisms when effective, but does not depend upon them to solve all problems.

- $\quad$ Has a standard of values that guides actions.

- Is able to change or adjust behaviour when necessary, but only if moral standards are maintained.

- Has developed a philosophy of life that meets the demands of society, satisfies personal dreams and goals, and is within his/her capabilities.

- $\quad$ Can control anger and hostility, as well as other emotions, and has learned harmless ways of getting rid of hostility.

- Develops creative interests and abilities so that satisfaction can be found in doing things well.

- $\quad$ Takes pride in being prepared to accept responsibility for ideas, feelings, and actions.

\section{Step Towards Imbibing Mental Health By Every Individual}

It is very essential for every individual to aim at positive mental health. Positive mental health, according to Isaac (2013), is a state of wellbeing that allows people to live up to their abilities, cope with the normal stresses of life, work productively to make a contribution to their communities, and establish healthy relationships with people.

Developing and promoting mental health services at the grassroots level and also maintaining a global perspective is an overwhelming task according to Schweiger and Hinkle (2013). Murthy (2006) indicated that there is no global community mental health blue print to achieve universal mental health access, and that, effective community workforce strategies need to be matched to each country's unique situation. It is an ecological fallacy to try to understand people and mental health issues outside the environments in which they exist. For this reason, a radical shift is urgently needed in the way mental disorders are managed. Hence, this clearly includes a community base care that can be effectively implemented through non-health as well as health sectors.

Implementing grassroots community mental health programmes is a step towards encouraging people to imbibe mental health. It may not be easy, but the global health care organizations must demonstrate greater need to develop innovative use of informal mental health assistants and facilitators to establish community mental health services. The programme in mental health consists of integrated knowledge ranging from basic mental health information and promotion to specific local and culturally relevant helping 
strategies (Furtos, 2013). Moreso, the programme provides equitable access to quality first contact interventions, including mental health advocacy, helping skills, monitoring, and referral. All of which respect human dignity and rights, amongst others. These assist in meeting local population needs.

Mental health is crucial to individuals, families, relationships, societies, communities, economies, and countries. The benefits of mental health to individuals include; adding to personal confidence, improving communication skills, enhancing personal relationships with others, and improving career opportunities. Good mental health is required for individuals' success and achievement in life. The parents, teachers, public, students, amongst others, ought to be familiar with mental health problems. Creating this awareness and imbibing of mental health culture would enable the people to develop preventive measures that could possibly help them handle and prevent future health problems.

Another procedure through which mental health can be imbibed by individuals is through teaching and learning. Mental health is effectively achieved through teaching and learning processes. This is enhanced by learning the skills in mental health and focusing on proper development of personality. In this respect, the schools, the community, health personnel, mental health facilitator, amongst others play a major role in the preservation and promotion of mental health of the students and members of the community. In the same vein, the school, community, and other stakeholders must provide a suitable emotional, intellectual, moral, and physical environment in which the child and adults have the feelings of security and belongingness.

There is an adage which says health is wealth. This is true because wellness involves one's physical as well as mental health. One can enjoy good mental health only when one is cautious about his/her mental health and know its value along with the knowledge and readiness in imbibing it, adopting its skills, and maintaining it. Good mental health is therefore very important to every individual's successful living and proper adjustment to life.

Therefore, other ways of imbibing good mental health include:

- $\quad$ Get ready for adoption of mental health skills.

- $\quad$ Learn about your environment

- $\quad$ Learn about the people around you.

- $\quad$ Learn about the problems.

- $\quad$ Consider various solutions

- $\quad$ Help others to achieve a solution or connect the person to resources.

- $\quad$ Follow up, among others. 


\section{Value Clarification}

It is commonly known that top-down approaches across public services generally have limited success. Conversely, long term strategies that enhance the successful implementation of public mental health services are best when they are centrally facilitated from a locally directed, bottom - up approach. According to Rock, Combrinck and Groves (2001), a service delivery and administrative perspective of mental health services must be addressed.

Partnerships among NGO, government, agencies and schools can make a difference in the mental health workforce capacity training by integrating global expertise with local knowledge. As many countries now recognize the in-depth of community health services. They also attempt to develop fundamental services in line with mental health focus. The Mental Health Facilitator (MHF) with their training program could be of great assistance.

According to Hinkle and Henderson (2007), the MHF curriculum is based on the universality of mental stress, distress, and disorders. MHF training includes numerous topics such as; basic helping skills, coping with stress, and community mental health services. The program consists of fundamental integrated mental health knowledge and skills ranging from community advocacy and commitment to specified interventions such as suicide mitigation.

Human beings are created specially, such that they must take cognizance of their physical health and surrounding environment to enhance good mental health. Good mental health is composed of healthy body, thinking clearly, sound mind, and getting well along with other people. Mental health knowledge is creating people awareness to health care and health sanitation at home and at school. It is also very important according to Hinkle (2010) to help people know the difference between harmful and healthful behavior. A healthful behaviour is an action taken to be a healthy eating in a healthful behavior, exercising a healthful behavior, keeping the environment, and relating with people in a healthful behavior.

Rafferty (2008) listed some healthful behaviours as management skills, life management skills, and coping with people management skills. However, these are mental healthful behaviours used for learning and to continue throughout life.

\section{The Need for Mental Health}

The core concept of mental health is consistent with its wide and valued interpretation across cultures. Mental health is determined by socioeconomic and environmental factors with which people are interacted and integrated into human life pattern. Mental health and mental illnesses, 
according to Okpenge (2014), are determined by multiple and interacting social psychological and biological factors. This is just as health and illness in general with the above assertion. It is evident that human being cannot do without mental health. Mental health is a blessing we must grasp and spread to other people. It is not just something we get once and we forget; mental health needs to be well taken care of at all times. However, mental health is not just the absence of mental disorder, but a state in which a person is able to fulfill an active functioning role in the community and interacting with others and overcoming difficulty without suffering major distress, abnormality, or disturbed behaviour. As such, the people need to be counseled to be able to maintain positive mental health and health promoting behaviours that could lead to individual mental health.

Maintaining good mental health is crucial to living a long, positive, and healthy life. Good mental health can enhance one's life, while poor mental health can prevent someone from living an enriching life. For these reasons, there is need for mental health knowledge to be allowed with good stress management, enabling physical health, emotional abilities, and prevent anti-social behaviours. Emotional mental disorders are a leading cause of disabilities worldwide. Investigating the degree and severity of untreated emotional mental disorder throughout the world is a top priority of the World Mental Health (WMH) survey initiative which was created by WHO in 1998. As such, there is need for prioritizing mental health.

According to Adedokun (2014), the 2004 WHO report "prevention of mental disorders" stated that the prevention of these disorders is obviously one of the most effective ways to reduce the (disease) "burden", which is through inculcating the mind for mental health. The 2011 Mental Health Strategy of Manitoba (Canada) included intents to reduce risk factors associated with mental ill-health and increase mental health promotion for both adults and children.

A mentally healthy individual can be easily distinguished from others by his mode of living, behaviour, dressing, mental abilities, capabilities, and his personality characteristics; as such, there is the need for mental health reorientation for all categories of people. For one to be mentally healthy, one needs to develop mental healthy skills. That is, learn many facts about mental health, learn how to use these facts, learn how to turn the facts into actions. These healthy and responsible actions are referred to as life management skills.

\section{Why Mental Health?}

Mental health allows one to feel good about oneself and the surrounding environment. When an individual knows that he/she can do 
something well, can think well, and relate well, he/she has a good selfconcept. Self-concept is the feeling that one has about him/herself.

\section{Economic and Health Implication of Mental Health:}

Mental health stands for the health of the mind and it functions in the same way as the physical health is concerned with the health of one's physical organs and their functions . According to Hinkle (2010), mental health services are inequitably distributed. Lower income countries where behavioural risk factors tend to cluster among people of lower socioeconomic status have significantly fewer mental health human resources than higher income countries.

In low to middle income countries, human resources are clearly limited, and the quality and productivity of existing workforce is often challenged. Investment in human resources for community mental health care, according to Hongoro and Mcpake (2004), is insufficient in absolute terms as well as in distribution.

Subsequently, there are two facets for integrating mental health into primary care; (a) financial and human resources (b) collaboration with nonhealth sectors. Unfortunately, governmental psychiatric hospitals have a long history of human rights violations and poor clinical outcomes; they are also costly and consume a disproportionate amount of mental health care money (Hinkle, 2010). Despite the array of treatments for mental health, little evidence exists regarding their feasibility and effectiveness when integrated into routine care settings among low and middle income countries. According to Becker and Kleinman (2013), the government of low income countries was constrained by lack of resources. In fact, in $85 \%$ of low income countries like Nigeria, essential psychotropic medications are not available. Money for mental health care is disproportionately lacking even though their associated burden is tremendous.

It was also discovered that any attempt to adapt to the continued presence of a stressor, body, and environmental hardship may deplete both the bodies and mental resources and make people vulnerable to serious illness. Too much of uncared health issues can have negative effects on human health, contributing and leading to so many problems including; heart disease, high blood pressure, stroke, depression and sleep disorders. Good mental health is required for an individual's success and achievement in life. Every parent, guardian, young and old ought to be familiar with mental health problems. This allows adequate preventive measures to be enhanced.

Mallum (2005) said that man is basically co-operative, constructive, and trustworthy. This indicates that, man is capable of relating with himself so that, he can move from unpleasant state to a well-adjusted and healthy 
state. In other words, man if provided with the required therapeutic relationship, will be able to solve his problems and live a balanced life.

Every person likes good health and every person wants to live a healthy life. Yet, sometimes we fall sick. Diseases disrupt the normal functions of the body and affect a person's normal work and social activities. Diseases also affect the economy of families and nations. Sick persons may not be productive at work, but may spend needed money on hospital bills and treatment. Diseases can be prevented or controlled through good mental health education and mental practices.

\section{The Need for the Re-Orientation of Nigerians on Mental Health}

Orientation means trying to direct or provide value or guide for an action. Re-orientation, on the other hand, means to re-educate about something or about a situation. It is a process of changing the old and inappropriate value orientation of an individual to enable him/her appreciate and acquire new set of values or ideas that are better off

The need to address workforce issues affects the quality and quantity of international mental health services. Therefore, there is a clear link between human resources and population health. Community and family needs to be sensitized and adequately adopt good mental health. This really calls for the need for urgent development of grassroots community mental health program. Community mental health workers must realize and know that a key component of any program must have that nature of flexibility. Flexibility allows for the modification and contextualization of programmes by local leaders to reflect realities of current social contexts and circumstances (Furtos, 2013).

Becker and Kleinman (2013) stated the principle that mental health care is a combination of basic, universally applicable context with specific knowledge and skills. Supportive social networks in the community pertaining to mental health could enhance wide knowledge among individuals about the essentiality of living healthy.

To facilitate better re-orientation and appropriate change, the under listed situations must be established:

- Investigate what is happening now that the individual wants to change.

- $\quad$ Summarize the situation or problem the person finds troubling.

- $\quad$ Ask how things could be different.

- $\quad$ Get a clear picture of the problem with specific details.

Many of the known diseases caused by micro-organisms can be prevented by good mental health sensitization. This could be effectively done through:

(a) Sanitation 
(b) Health education

(c) Immunization

(d) Pre-marriage screening

(e) Immediate treatment of infected person

\section{(a) Sanitation}

According to Rafferty (2008), sanitation involves the practice of maintaining clean and healthy environment. Some diseases spread through poor sanitation, and such diseases can be controlled by good sanitation. The re-orientation of the public towards good sanitation helps to remove from home surroundings rotten organic matters, stagnant water, refuse, and sewage that provide living places with germs. Ensuring good sanitation will prevent contamination of food and water which could help to control the spread of diseases like cholera and other communicable and noncommunicable diseases.

\section{Personal Hygiene}

This includes washing of the body and clothes regularly and proper clearing of parts of the body. Rao and Raju (2012) emphasized that good personal hygiene rescues the body from all forms of deadly diseases and other illnesses.

\section{(b) Health Education}

According to Lewis (2011), some people lack the awareness of the causes of diseases, how they spread, and how they can be prevented. Educating people about these will create the awareness and enable such individuals to take preventive measure against contracting diseases. Health education, therefore, is an important means of enhancing good mental health because it assists the prevention of the spread of diseases.

\section{(c) Immunization}

Immunization is a method of preventing diseases by injecting into the body specially treated diseases-causing organisms or their products. Lewis (2011) said that when such materials enter the human body, the body reacts as if the disease-causing micro-organisms are actually present, but the person does not fall ill. The body produces substance, called antibodies, which are carried in the blood. The antibodies remain in the body and fight against disease-causing microorganisms that may likely attack the body (Lebowitz \& Omer, 2013). Furthermore, the public needs to be orientated on the importance of immunization which is to stimulate the body to produce antibodies against some diseases that can be deadly to human body.

The government of Nigeria in collaboration with the World Health Organization (WHO) and the United Nations International children's Fund (UNICEF) emphasized on constant advocacy programs on seven diseases 
that commonly cause death: Cholera, tuberculosis, tetanus, yellow fever, measles, whooping cough, and diphthewa (Pijl, 2010).

\section{(d) Pre-marital Medical Screening}

Indiscriminate sexual relationship creates high risk of being infected with sexually transmitted diseases including HIV/AIDs. The need for training and re-training about the choice of a life partner should be critically considered based on the health status of each. The prospective couples should be informed on the needs to carryout diseases and blood-screening test such as HIV/AIDs, genotype, Haphes among others before agreeing to marry each other. Screening creates awareness to hereditary and sexually transmitted diseases and how they can be avoided.

\section{(e) Counseling Implications of Mental Health}

Counseling is a form of assistance that involves many activities that will help individual in understanding him or herself and the problems encountered.

Therefore, a lot of benefits would accrue when mental health counseling is adequately inculcated into public health programs to include;

- $\quad$ Developed health skills, self-management skills, and self-discipline culture useful in maintaining a positive and stable emotional and sociopsychological well-being.

- $\quad$ Identify health practices and cultures that will shape and encourage the people now, in future, and their future health life styles.

Failure and frustrations in life are signs and symptoms of poor mental health which may be situational, but which may also be resolved through counselor's regular advocacy, sensitization mental health programmes, and health monitoring programmes.

Stress copying, good diets, regular exercise, good living condition, among other strategies should be taught to students and entire society by the counsellors through organized seminars and workshops.

Good mental health is a pre-requisite for good health living, good mental ability, emotional balance, and positive interpersonal relationship. As such, the counselor has a great role to play in the re- education of the incidence that could enhance effectiveness in the above listed factors.

The counselor should also assist every individual to develop his/her positive health self-concept. The counselor should ensure to sensitize the public with information about their lifestyle regarding sleeping procedures, eating behavior, nutrition, exercise, stress and its management, life coping mechanisms, blood screening and genotype tests habit, regular check-up among many others. This will go a long way to enhance good healthy living and individual's emotional and psychological balance. 


\section{Recommendations}

Behavior modification could be adopted not only to eliminate negative behavior among the people towards maintaining good mental health, but could also be used to develop and increase the people's social desirable behavior for their well-being, maintaining health living, and positive life-style.

The public health situation now and later should be of great worry especially considering the ravaging Sexually Transmitted Diseases (STDs), HIV/AIDs, increase abuse of drugs, mental problems, fever, behaviour problems among others that are threatening to wipe out the nation's potentials. As such, functional and pragmatic intervention programmes by the government are urgently required to checkmate the negative trend of these nasty problems.

The government in collaboration with the counselors and NGOs should embark on comprehensive good mental health preventive programmes. The effectiveness of these programmes will assist to reduce high risk problems experienced through lack of mental health information. Such information and mental health education could essentially decrease the number of people that are victims of various forms of health diseases and illnesses.

The three tiers of governments should embark on regular workshops, seminars, radio and television programmes, using various models, health experts, sociologists, counselors mental health facilitators, among others to carry information regarding good mental health to grassroots level in every state of Nigeria. This will go a long way to imbibe good mental health management skills in the people's daily activities. This will also reduce the huge amount the government spends on health problems and in the purchase of drugs yearly.

Mental health routine guidance teaching and information programmes dissemination should be embedded into all schools and institutions' curriculums and programmes. The government should employ more professional counselors into all levels of schools and colleges and must be encouraged to provide valuable information on good mental health behavior and its consequences.

\section{Conclusions}

It is evident from literature and information received that the public are not well prepared and they also lack adequate dissemination information about mental health. This has already killed vast numbers of people, and threatens millions more in the near future. A probable inference may not be unconnected to inadequate education relating to good mental health in a time like this when STD, HIV/AIDs, Fevers, Ebola, Hypertension, Diabetes, 
Cholera, and other health diseases threatens human existence. To get out of this glaring looming doom in the society, functional education by mental health facilitators and counselors is the panacea.

\section{References:}

1. Adedokun, R. (2014). Group Counselling Intervention in preventing mental health disorders among Nigerian undergraduates. Perspectives in Guidance and Counselling. 373-382.

2. Aggarwal, A. (2011). Impact of Academic Stress upon academic Achievement and mental Health of the Adolescents, International Journal of Management and Social Sciences, 1(1). Retrieved from http://www.ijmss.com.

3. Furtors, J. (2013). Globalization and Mental Health: The Weight of the World, the Size of the Sky. Presidential Symposium Conducted at the American Psychratric Association Annual Meeting. San Francisco (2007).

4. Hinkle, and Henderson, D. (2007). Mental Health Facilitation. Greenshome, NC: NBCC International.

5. Hinkle, J.S. (2010). Mental Health Facilitation: A global Challenge Workable Strategy: Paper Presented at the 18th APECA Biennial Conference-Workshop, Penang-Malaysia.

6. Hongoro, C. and McPake, B. (2014). How to bridge the gap in human resources for health the Lancet, 364, 145-1456. Do: 10.1016/80.

7. Isaac, M. (2013). Document two. In Jide Almeida (Chair), Innovation in deinstitutionalization: A WHO expert survey. Symposium conducted at the international forum on Innovation in Mental health, GulbenKran Global Mental Health Platform. Lisbon, Portugal.

8. John, A.F. (2012). Mental Health Literacy: Empowering the Community to take action for better mental health. American Psychologist, 67(3), 231.do:10.1037/a0025957.

9. Kleinman M.D. (2013). Towards a public mental health promotion and interventions system for youths. The Journal of School Health, 71, 101-105.

10. Lebowitz, E.R. and Omer, H. (2015). Treating Childhood and adolescent anxiety: A guide for care nears. Hoboken, NJ: John Willey.

11. Lewis, C. (2011). Providing therapy to children and families in foster care; A system relational approach. Family process, 50(4), 436-452, do: $1111 / \mathrm{J}$.

12. Mallum, M.P. (2015). Guidance and Counselling Services in Nigeria. Jos: Yabyangs Publishers. 
13. Murthy R.S. (2006). Mental Health by the "People, "Peoples action for Mental Health, Bangalore, India.

14. Nordquist D. (2015). Crisis counseling assistance and training programme: practices document, American Association for Marriage and Family Therapy.

15. Okpenge, E.G. (2008). Guidance and counselling: A sine qua non to Mental Health Multidisciplinary Journal of Research Development. $6(2)$ 52-55.

16. Orleans B. (2014). Effective Helping: Interviewing and Counseling Techniques. Pacific Grove, C.A.

17. Patel, V. (2013). Integrating Mental Health care in Priority Health Programmes. Addressing a Grand Challenge in Global Mental Health, Manuscript Presented at the International Forum in Innovation in Mental Health, Gulbenkin Global Mental Helath Platform, Lisbn, Portugal.

18. Pijl, S.Z. (2010). Preparing Teachers for inclusive education: some reflections from the Netherlands of Research in special education needs, 10(51), 197-201, do: 1111j: 1471-3802-2010.

19. Rafferty, Y. (2008). The Impact of Trafficking on Children. Child Development Psychological and social Policy Perspectives. 2(1) 13 18.

20. Raw, K. and Raj. U,M. (2012). Early Adolescent. Emotional and Behavioural Problems. Journal of the Indian Academy of Applied Psychology, 38(1) 34 - 39.

21. Rock, D. Combrinck, J. and Groves, A. (2001). Issues Associated with the Implementation of Routine Outcome Measures in Public Mental Health Services. Australasian Psychiatry, 9(1), 43-46.

22. Sustainable Development Goal (SDG) (2015). Health Focus and Community Development.

23. WHO (2000). Preventing Suicide: A resource for Media Professionals. Geneva, Switzerland Author.

24. WHO (2001). World Health Report Geneva, Switzerland: Author.

25. WHO (2006). Integrating Mental Health into Primary Care: A Global Perspective. Geneva, Switzerland: Author.

26. WHO (2010). Mental Health and Development: Targeting People with Mental Health Conditions as a Vulnerable Groups. Geneva, Switzerland: Author. 